\title{
Research on the Cognitive Effort of Sight-Interpreting Complex English Sentences into Chinese: Evidence from Eye Tracking
}

\author{
Mengsheng Qian ${ }^{1}$, Ningjun $\mathrm{Xu}^{2,}$ * \\ ${ }^{1}$ School of Foreign Languages and Cultures, Nanjing Normal University, Nanjing, PR China \\ ${ }^{2}$ College of Humanities and Management, Zhejiang Chinese Medical University, Hangzhou, PR China
}

Email address:

judexu@zcmu.edu.cn (Ningjun Xu)

${ }^{*}$ Corresponding author

To cite this article:

Mengsheng Qian, Ningjun Xu. Research on the Cognitive Effort of Sight-Interpreting Complex English Sentences into Chinese: Evidence from Eye Tracking. International Journal of Applied Linguistics and Translation. Vol. 6, No. 4, 2020, pp. 116-123. doi: $10.11648 /$ j.ijalt.20200604.12

Received: December 6, 2020; Accepted: December 18, 2020; Published: December 25, 2020

\begin{abstract}
It is widely known that sight-interpreting, one of the typical forms of conference interpreting, requires the interpreters to exert great effort in transforming one language into another. Due to the difference between Chinese and English, some sentence structures such as relative clauses prove to be even more difficult to render. Some experienced interpreters are able to do such strenuous task with ease. Uncovering what is going on during information processing is enlightening in that it would shed light on how human brain uses certain types of mechanism to process information, which is conducive to the artificial intelligence. Eye-tracking experiment is designed, 31 subjects are recruited with an average age of 22 and comparable linguistic competence to participate in a 40-50 min experiment, during which each subject is required to sight-interpret the self-designed, expert-proven sentences which differ only in the role of the relative pronoun in the relative clauses. Data analysis clearly indicates that the cognitive effort of processing complex sentences as evidenced by two types of relative clauses (one is called OR because the relative pronouns function as object in the relative clause, the other SB because the relative pronouns function as subject in the relative clause) are different, the former requiring more cognitive effort than the latter, as shown in several key eye-movement measures such as regression-in, regression-out, first fixation duration, gaze duration, regression duration, and total reading duration. These differences are statistically significant within the AOIs such as the antecedent, relative clause. The finding further substantiates the hypothesis that sight-interpreting is more strenuous and thus requires more cognitive effort than the common readers. Besides, different structure of the relative clauses also plays a role in consuming the cognitive effort of the interpreters. However, it remains unclear whether the length of the relative clause plays a decisive role in influencing the cognition of whole sentence while sight-interpreting. Besides, whether the research results are applicable to other types of complex structure remain unanswered. More data should be collected to incorporate more complex structure in order to uncover the possible cognitive effort during sight-interpreting.
\end{abstract}

Keywords: Cognitive Effort, Sight-interpreting, Complex Sentences, Relative Clause, Eye-tracking, Eye-movement

\section{Introduction and Literature Review}

In the process of transforming concepts encoded in one form into another, a lot of efforts have to be exerted. Broadly speaking, the process of such transformation can be roughly divided into two categories in terms of linguistic phenomenon. One is intra-linguistic transformation, involving the rendering of the concepts across the historical record of a particular language, e.g., from Old English to Modern English and vice versa. The other way is rendering the presentation of concepts across different languages [1]. No matter in which way the transformation is involved, it at least can be approached either through written translation or oral rendition, the former being translation while the latter interpretation. There are numerous ways of research into translation as it played and will still play 
a definitive role in facilitating the cultural transmission of human intelligence. The underlying reason is quite straightforward. First of all, written translation is not instantaneous. In other words, the interpreter has plenty of time weighing the different versions before the final draft can be identified. The best translation is made possible out of the proofreading by different editors. One can never claim that the final version is not the collaboration between the interpreter and the editor (s) though the editor (s) would seldom become the co-interpreter. Besides, for lack of time and space constraints compared with interpretation, written translation can be done in a relatively light and comfortable environment. It is claimed that anxiety out of time deadline is a major factor influencing the quality of transformation [2]. In line of the two factors, it is comparatively speaking, easier to do research into written translation than oral interpretation in terms of how the brain of the interpreters works when they are doing translation.

However, oral interpretation has drawn the attention of researchers recently mainly due to two reasons. Because of the development of artificial intelligence, scientists are interested in discovering how human brain works under pressure especially in terms of concept presentation. If it is possible to identify the neural process of conception transmission, then such process can be simulated and imitated by machines which finally could simulate human brain, thus the birth of true human intelligence-possessed machines. The research into the process of interpretation is relevant because it would uncover the possible link between different languages [3], one of which could be machine language. Scientists could then design the information processing link between humans and robots through applying such neurol interconnectivity. Similarly, the underlying mechanism could also be applied to the communication between machines empowered with certain intelligence. The fast development of neuroscience, especially in the field of medical technology, makes it possible to probe into human brain to find how each area of the brain interacts one other [4]. However, so far, it is still relatively unknown as to how the advanced cognitive activities such as conception transformation is processed in the brain. It is widely acknowledged that oral interpretation is arduous due to its time and space constraints, thus making it a highly advanced cognitive process. The research into oral interpretation would shed light on how the brain of some higher species such as humans cope with intellectual emergency.

Roughly, there are two ways that the source language can be input during interpretation. One is through listening, thus the conference interpretation and consecutive interpretation. The other is through visual input, thus the sight interpretation. Considering the fact that conference interpretation is rather sophisticated and is involved of many steps in cognitive process, the sight interpretation is chosen as a preliminary probe into the mysterious world of oral interpretation. There are at least three advantages of using sight interpretation as a representative of doing research of oral interpretation. First, sight interpretation is widely acknowledged as a good way of indicating the capability of a qualified interpreter's performance. Research shows that sight interpretation requires basically the same amount of effort as other types of oral interpretation [5]. Therefore, coping with sight interpretation is at least as demanding as conference interpretation. A second advantage involves the time invested in sight interpretation. It is as time immediacy as other types of interpretation in that the former requires the interpreter to render the possible version as soon as he/she begins reading the source language. Lastly, it is relatively easier to manipulate the subjects during experiment. In other words, in order to design a research experiment, researchers tend to simulate the reality as much as possible. Due to the complexity of conference interpretation, it is nearly impossible to duplicate the working environment under which a professional interpreter does the conference interpretation. However, creating a suitable atmosphere for interpreters to do sight interpretation is possible and rather simple: giving him/her a sheet of paper, then the interpreter can begin to work. It can be done in a language process lab by replacing such piece of paper with the computer screen, on which the desired language can be manipulated in order to test and uncover the possible working mechanism of the cognitive process when an interpreter is doing their job.

Since the turn of the $21^{\text {st }}$ century, because of the development of cognitive science, academic circles had begun to pay attention to the analysis of brain cognitive processing in interpretation [6]. The purpose is quite straightforward. If the interpretation skills and methods possessed by professionals can be explored and illuminated in terms of cognition, then training young interpreters would become easier. Since so many researches have been done between Chinese and English in the field of interpretation, one particular linguistic difference attracts our attention. English and Chinese roughly speaking follows the same pattern of sentence structure: SVO, i.e., subject plus verb plus object. There are many different types of complex structures in English and Chinese. However, one particular structure in English, relative clause behaves rather differently in Chinese. In English, a relative clause normally occurs right after its antecedent; occasionally the antecedent and its relative clause is separated by several inserted words or phrases. Technically, Chinese lacks the same structure. It is rather conventional for Chinese to use an independent clause rather than embedding this clause into the structure [7]. Several strategies are proposed to cope with this interlinguistic transformation. The adoption of such strategies as sentence segmentation and sentence adjustment during sight interpretation adds to the difficulty of the interpreter's memory load and perception. Besides, there are two types of relative clause. One involves the subjective role played by the relative pronoun in the clause, the other the objective role. The purpose of doing this classification is that in the former situation, the relative pronoun is right after the antecedent while in the latter, the relative pronoun is distant from its antecedent. These two types of pronoun revolution prove to be different in the demand of cognitive effort. While the effort made to resolve the short distance anaphora is not much, the interpreter must pay much more efforts in order to find the 
reference of relative pronouns. Traditionally, the result of such resolution depends on personal report, which would seriously damage the rigor of the research. One way of increasing the reliability of the data is through eye-tracking technology, an increasingly popular and reliable way of probing into the brain activity through eye-movement tracking. Therefore, in order to truly uncover the possible mechanism of sigh-interpreting, this article will try to explore the cognitive processing of complex sentences as evidenced by relative clauses in the English-Chinese sight interpretation under the framework of eye movement experiments, hoping to provide some possible enlightenment for the study of interpretation process.

The sight interpretation in this paper is defined as a process in which subjects produce an interlinguistic output from English sentences present on screen to Chinese at their own speed. Not so many researchers focus on how the interpreters manage to output the target language. So far, the earliest interpretation process model was put forward by French scholar Gile [8], who claimed that the whole process of sight interpreting consists of two steps: reading analysis and verbal output. This is rather straightforward and quite powerful. It is straightforward in that the whole process indeed can be divided brutally into two parts, reading and interpreting. But do these two steps separate each other or are they overlapping to some extent? Is there something else that takes place besides reading and interpreting? It is accepted nowadays that reading and interpreting sometimes are overlapping a great deal and there are more than two steps involved in sight interpreting. Wan Hongyu [9], for example, enlarged the simple model: sight interpretation $=$ reading + memorization + coordination + interpreting output. This model adds two important cognitive steps -memorization and coordination. Other scholars proposed different models from different perspectives. Wang Jianhua [5] concluded that the optimum model of extracting information is title plus frequent expressions during interpreting narratives while the best summative information in an explanatory essay is in its first sentence plus frequent expressions. Deng Wei [10] identified six strategies that can be employed in sigh interpreting, such as sentence-order based interpreting, stopping at proper places, conversion of part of speech, strategies coping with relative clauses and passive sentences, and so on.

As a common example of complex English sentence patterns, relative clauses, which are dependent on the relative pronouns, can both act as subjects (hereinafter referred to as SR relative clauses) and object (hereinafter referred to as OR relative clauses) in clauses. Chen Dezhang [11] argued that relative clauses are frequently used in English language, yet unknown in Chinese language. It is called as "attributive clause". Yet, semantically, relative clause sometimes does not provide qualified information to the nearby sentence. More often than not, relative clauses provide more information, sometimes additional, sometimes restrictive, to the main clause. Different strategies do exist in handling these two different situations. In terms of English syntax, the logical connection between the antecedent and its relative pronoun can be about reason, result, condition, purpose, concession, time, etc. [12]. However, so far, no paper is contributed to the study of interpreting of relative clause from a cognitive perspective, let alone classifying relatives into SR and OR. Studies have shown that English-speaking readers need more cognitive effort when reading OR relative clauses [13]. Based on this, it can be assumed that the different grammatical roles relative pronouns play would affect the understanding of the source language and thus influencing the output of the target language during English-Chinese sight interpretation. Therefore, the research question raised in this paper is as follows:

How much cognitive effort do interpreters have to exert in order to render a satisfactory output during sight-interpreting? Specifically, is there any difference in processing different structures of complex sentences such as SR relative clauses and OR relative clauses?

Based on the special syntactic nature of relative clauses [12], a preliminary hypothesis is put forward: during sight interpretation, there exist some differences in the degree to which the two types of relative clauses affect the understanding of the original text.

\section{An Introduction to Eye Movement Technology}

During the research of relative clauses in the field of cognitive science, eye-tracking technology has played a key role in uncovering the possible mechanism between the processing of information and verbal output. In other words, if there exists a close connection between the process of sight interpretation and the psychological process of interpreters, and if researchers desire to unravel this mystery, they have to resort to modern science and technology, such as eyeball-tracking technology. Based on the "eye-brain" hypothesis whose core is that the point of attention is the point of information processing [14], eye movement technology can depict the state of eye movement of interpreters during sight interpretation and reveal the instant on-line cognitive processing of the subjects while they are processing relative clauses by recording relevant parameters.

The working mechanism of eye-tracking technology is quite simple. During reading, the eyeballs are jumping forward and backward, sometimes stop at a particular place of interest. It is said that the information process only takes place when the eyeballs are fixed on a particular place. Jumping from place to place does not involve any cognitive processing [15]. In this way, different patterns of jumping and fixating indicate different processing methods and cognitive load. In the long history of eye-tracking technology, scientists have identified three basic modes of eye movement: fixation, saccade, and regression. Fixation, which is the action of focusing on information within the fovea of the eyeball for more than 100 milliseconds, is the main method of obtaining and processing information. Saccade refers to the continuous movement of the eyeball and no information can be obtained during this process. Regression refers to the eye movement of 
looking back at the previous text, which is helpful for deep processing [14]. Rayner [16] argued that the reason why readers are doing regression is due to the fact that the fixation is on the wrong word or that the reader might not understand what is going on or he/she is confused about what is being read therefore requiring re-reading to affirm, to deny, to re-process the past information. Meaningful collection of relevant data during interpreting would help to posit effective psychological patten of the interpreters during working.

\section{Design of Experiment}

Since the analysis of information processing, to a great extent, depends on how the brain uses neural connectivity to transmit energy, thus serving as a way of information exchange, the possibility of uncovering the underlying mechanism is realized by exploring the relevant link between eye movement and the place of interest. As the distance between the anaphora and reference influences the processing mechanism and outcome of pronoun revolution, it is necessary to design an experiment which could reflect the difference only in one aspect, i.e., distance while the rest remains the same. The research question in current paper is to explore the possible mechanism of processing two types of relative clauses from English into Chinese during sight interpretation. Therefore, there are at least one independent variable, i.e., whether the relative clause is SR structure or OR structure, more specifically, the relative clause only involves the relative pronoun of "who", which in daily English, could act both as subject and object in relative clauses. Thus, this experiment uses a single-factor within-subject design with two levels, and the variable involved is two grammatical role of the relative pronoun "who" in the relative clause.

\subsection{Selection of Research Subjects}

Altogether, 31 subjects from a renowned university have been recruited randomly through internet whose majors are Translation Studies (English). They have self-reported high English proficiency, all passed TEM4. Their competence of sight interpretation is of the average, none of whom have received intensive training in either type of interpretation. It is also worth mentioning that they all possess a second language competence, such as Japanese, French, German. This additional linguistic competence might influence the result of subjects' performance in sight-interpreting but is not considered as a decisive role in data analysis.

Other parameters including age, eyesight, right-handedness are basically not varied, all within 20-23 years of age, 1.0-1.5 original or corrected eyesight, and right-handed. All except one subject have participated eye-movement experiment; none of them have taken part in the sight-interpretation experiment.

\subsection{Experimental Materials}

All experimental materials are self-made, and the vocabulary used comes from BNC corpus of 4000 high-frequency words. The experimental materials include 80 sentences serving as experimental materials, 36 sentences serving as fillers and 8 sentences as pre-experiment exercises. The level of difficulty and readability of all sentences were assessed by five native English-speakers.

After the data was prepared, an independent group of subjects were recruited to pretest the validity and reliability of the experimental materials. These subjects shared the same parameters, including linguistic competence, age, right-handedness, eyesight, etc., and all signed confidential contract to make sure no information about the experiment would be leaked. The pretest rendered 8 sentences unfit for experiment, thus being excluded from the final data. Therefore, there are 72 sentences used in the experiment.

\subsection{Procedure of Experiment}

This study adopts the classic eye-tracking paradigm of single-sentence appearing once at one time in the middle of the computer screen. The experimental equipment is Eye-link II. The experimental operation was carried out with the built-in software. The subjects were sitting on the armchair in front of the monitor with their heads rested on head-supporters, and their eyes were about $50 \mathrm{~cm}$ away from the center of the screen.

Three-point calibration method was used to make sure the eye-tracker could successfully track the pupil of the subject with satisfactory accuracy. The experiment process is as follows: 1) each subject is required to fill in the information form, indicating necessary background data about the subject as discussed in the subject sector; 2) after sitting in front of the computer screen, the subject received experiment training, during which the purpose and method of data collecting was told to the subject by an independent operator rather than the researchers of this paper in order to avoid any possible subjective interference; 3) after successfully passing the training session, the subjects engaged in formal experiment. The experimental sentences were presented randomly in the screen. The subjects sight-interpret them sentence by sentence. They can wait as long as they wish when the sentence appeared on the screen. After one sentence is finished, the subject presses any key to continue. The designed the pause is roughly between 30 and 32 sentences. But the subject can ask for a pause as long as she/he wishes. The purpose of pauses is to make sure the calibrated accuracy can be maintained to a satisfactory level. The average duration of experiment of each subject lasted between 40 to 50 minutes.

\section{Analysis of Data and Discussions}

The experiment was done during the late autumn and early winter of 2019 in the laboratory of a key university in China. After the data was collected, the original data underwent standardized process by which the abnormal data was eliminated from the corpus. On the whole, three datasets were eliminated for incompleteness of recording. The following part is devoted to the discussion of sight-interpreting of the relative clauses from global and 
regional areas of interests which will be defined in the next section.

\subsection{Analysis of the Data About Sight-interpreting the Whole Sentence}

In the process of data analysis, researchers need to determine the target area according to the research purpose, viz. Area of Interest (AOI). In order to explore the cognitive efforts of the two types of relative clauses, the researcher divided the whole sentence into four AOIs, viz. antecedent, relative clause, subject verb, and other parts. The data collected while the subjects were reading other parts is ignored from the analysis because these parts do not influence the process of sight-translation of relative clauses.

Research [16] shows that three eye movement measures are critically important in showing the possible cognitive effort, i.e., mean fixation duration, number of fixations and total fixation duration. In the part of the sight-interpreting of the whole sentences, the differences between number of fixations and total reading duration were statistically significant except mean fixation duration (Table 1).

Table 1. Data of eye movement while sight-interpreting the whole sentence.

\begin{tabular}{llll}
\hline & Mean Fixation Duration & Number of Fixations & Total Fixation Duration \\
\hline OR & $321 \pm 8.4$ & $94 \pm 5.2$ & $30174 \pm 742$ \\
SR & $312 \pm 7.3$ & $78 \pm 3.4$ & $24336 \pm 607$ \\
$\mathrm{t}$ & $0.27(\mathrm{p}>0.05)$ & $2.43(\mathrm{p}<0.05)$ & $0.93(\mathrm{p}<0.01)$ \\
\hline
\end{tabular}

The mean of duration of all fixations in an AOI is the mean fixation duration. T-test shows there is no difference of statistical significance, that is, when the interrogative pronoun "who" serves as an object or a subject in a relative clause, it does not affect the mean fixation duration of the whole sentence. This is slightly different from the research of Gibson and his colleagues [13]. The reason may be that the interpreter pays attention to not only the clause itself, but also the verbs and other components after the clause. This is also in line with the global processing model put forward by Gile [8] who argues that the whole process of interpreting must involve at least memorization of almost whole sentence. Because there is no relative clause like English in Chinese, interpreters need to implement strategies such as conversion of voice and adjustment of word order [12], which can produce more fixations and weaken the influence of grammatical position of relative pronouns. The after-experiment interview with the subjects also proves it is possible, at least from the self-report reflection of sight-interpreting of each subject, that the subjects sometimes thinks too much while interpreting either OR or SR.

Number of fixations refers to the total number of fixations in a certain focused area [15]. This measure can effectively reflect the cognitive processing efforts of reading and analyzing materials. The more cognitive efforts of reading materials are, the more numbers of fixations are. T-test shows that the difference between these two types of relative clauses is statistically significant because p-value is less than 0.05 , namely, the number of fixations of the OR relative clause exceeds that of the SR relative clause. This shows that the former one requires interpreters to pay more cognitive efforts. It is within the commonsensical understanding of the difference between the SR and OR. After all, the interpreter must figure the long-distance anaphora between the antecedent and the relative pronoun though physically they appear side by side. In an OR sentence, the relative pronouns would appear at the end of the relative clause, increasing the cognitive effort exerted by the interpreters.

Total fixation duration, also known as total staying duration, refers to the total sum of fixations within a particular AOI [17]. T-test shows that the difference between these two types of relative clauses is statistically significant because $p$-value is less than 0.05 , namely, the total reading duration of the OR relative clause exceeds that of the SR relative clause. This shows that the former one has a greater influence on the time for interpreters to read original texts. In other words, the role of the relative pronouns does play a decisive role in figuring out the possible link between the pronoun and its antecedent, thus increasing the cognitive effort of the interpreters.

\subsection{Analysis of Data while Sight-interpreting Antecedent}

Although antecedent is not a part of relative clause, it is an indispensable element in semantic and syntactic processing. The whole existence of the relative clauses depends, to a great extent, on the role of the antecedent. The reason is quite simple. The antecedent would reappear itself in a relative clause, but only in the form of a relative pronouns. Successful interpreters are required to figure out such connection within a very short period of time and to render the possible interpretation at the same time. It is thus expected that eye-tracking measures in the area of antecedent would shed light on how much the interpreter's cognitive effort is utilized. Because the relationship between relative pronoun and antecedent belongs to cataphoric reference, regression-in and total reading duration directly show the processing effort of antecedent (Table 2).

Table 2. Data of eye movement while sight-interpreting antecedent.

\begin{tabular}{lll}
\hline & Regression-in & Total Reading Duration \\
\hline OR & $94 \%$ & $4917 \pm 731$ \\
SR & $73 \%$ & $3814 \pm 621$ \\
$\mathrm{t}$ & & $4.07(\mathrm{p}<0.01)$ \\
\hline
\end{tabular}


Regression-in refers to the ratio of the number of fixations of regression in the subsequent AOIs to the total number of fixations in that particular AOI. The higher the regression-in is, the more interest the interpreters have in that AOI and the lower the contextual predictability is [14]. The result of experiment shows that the antecedents in the OR relative clauses obtain more regression-in. In other words, the interpreter is trying to figure out the connection between the relative pronouns and their antecedents.

T-test of total reading duration shows that the difference between them is statistically significant because the p-value is less than 0.05 , that is, the total reading duration of the antecedents in OR relative clauses exceeds that of SR relative clauses. From the perspective of behavioral data, the reason may be that the long distance between relative pronouns and their antecedents contributes to the cognitive effort of interpreters when they judge the relationship between antecedents and other parts of relative clauses. This data is in an accordance with the native speakers' reading the OR and SR [13]. Once again, it proves that interpreting mechanism basically is similar to the pattern of native speakers' understanding of the original data.

\subsection{Analysis of Data While Sight-interpreting Relative Clauses}

There are a lot of ways to decipher the processing method of relative clauses while the subjects are reading and interpreting at the same time. Technically, researchers have found five key measure that can be used to indicate the processing of relative clauses: regression-out, first fixation duration, gaze duration, regression duration and total reading duration [16]. The dataset of each of the measures is as follows (Table 3 ).

Table 3. Data of eye movement while sight-interpreting relative clauses.

\begin{tabular}{llllll}
\hline & Regression-out & First Fixation Duration & Gaze Duration & Regression Duration & Total Reading Duration \\
\hline OR & $49 \%$ & $241 \pm 19$ & $1315 \pm 142$ & $1943 \pm 172$ \\
SR & $37 \%$ & $197 \pm 13$ & $1032 \pm 217$ & $1643 \pm 164$ & $8567 \pm 741$ \\
$t$ & & $-3.27^{*}$ & $4.75^{*}$ & $-6.29 *$ & $7032 \pm 621$ \\
\hline
\end{tabular}

*: All $p$-values are less than 0.01 .

Regression-out refers to the ratio of the number of fixation points of regression to the AOI to the number of fixation points in this AOI. It usually takes place under two situations either because the interpreter encounters a perceptive difficulty by skipping too many letters or because the initial perception of a particular word or expression is not fitting to the context. This indicator can convincingly reflect how the subjects discover difficulty of understanding in AOIs. The result of experiment shows that the regression-out of sight-interpreting OR relative clauses is higher than that of sight-interpreting SR relative clauses, which means that interpreters need to spend more time looking back at antecedents. This result is consistent with the research results that long-distance anaphoric reference needs interpreters' more cognitive efforts [18]. What seems to be more interesting here is that though the interpreters are trying to figure out what is going on in the linguistic context, it is also possible that the semantic relations among different constituents of the clause would give rise to regression-out.

First fixation duration generally refers to the fixation duration of the first fixation point of the subjects when they encounter the AOI in the first place. It is universally acknowledged that first fixation duration is a good measure to indicate the subjects' cognitive process of words in the early phase and the extent of their sensitivity of the difficulty in processing words [19]. The longer the first fixation duration, the more effort the interpreter might be exerting. It could also be possible that the interpreter is stuck in this place. T-test shows that the difference between them is statistically significant because the p-value is less than 0.05 , that is, the first fixation duration of OR relative clauses is more than that of SR relative clauses. The result shows that the cognitive efforts of OR relative clauses are more than that of SR relative clauses from the very start. In other words, the different structure of OR and SR, to a certain degree, influences the cognitive processing of relative clause during interpreting.

Gaze duration is also called dwelling duration of the first passage. It refers to the duration that lasts from the first fixation in the AOI to the time when the first fixation leaves this AOI [17], including the regression within AOIs. It is during gaze duration that the interpreting is processing and perceiving information. Once again, this measure validly indicates the amount of effort exerted by the interpreter. T-test shows that the difference between OR and SR is statistically significant because the p-value is less than 0.05 , that is, the gaze duration of OR relative clauses is more than that of SR relative clauses. Therefore, the cognitive efforts of interpreters while processing OR relative clauses are greater.

The last important measure of this experiment is regression duration, also known as reading duration of regression. It refers to the total sum of all the fixations that fall on the right side of AOI after regressing back and forth. Research shows that it is a useful parameter which can reflect the processing in the later phase [17]. Regression duration is able to tell the researchers how much time the interpreter uses in order to figure out the relation between what is past and what he or she is reading right at the moment. T-test shows that the difference between them is statistically significant because the $p$-value is less than 0.05 , that is, OR relative clauses need more regression duration, thus more cognitive effort. Obviously, the subjects are more liable to discover problems when processing OR relative clause, which means that they are more prone to re-read the previous part of sentence. The long distance between antecedent and its original place in the relative clause 
forces the interpreter to jump back and forth within the relative clause more frequently when they are processing OR relative clause than when they are processing SR relative clause. It is possible to explain that $\mathrm{OR}$ relative clause requires more strong working or short-term memory

As for total reading duration, t-test shows that the difference between them is of statistical significance because p-value is less than 0.05 , that is, the total reading duration of $\mathrm{OR}$ relative clauses is more than that of SR relative clauses. This shows that the cognitive efforts of interpreters while reading OR relative clauses are greater. After all, the reading time is a traditional and reliable indicator of the difficulty of a particular sentence. The longer the time used, the more difficult such sentences would appear. In sight-interpreting, the more time devoted to reading means the more time used to process the relevant information.

\subsection{Analysis of Data while Sight-interpreting Verbs in the Main Clauses}

The parameters of data while sight-interpreting verbs in main clauses are the same as those of relative clauses. Data of regression-out and regression duration differ significantly between SR and OR relative clauses. It could be possible to explain away the difference by attributing to the different structure as what is discussed in relative clauses. However, it could also be possible that the regression-out and regression duration is caused by the interaction between the verb and its adjacent structure. Therefore, it is so far indefinite as to the cause of such difference. More data should be analyzed in this area to determine how the verb in the main clause plays its role in influencing the processing of the relative clause.

Except regression-out and regression duration, there are no obvious differences in other measures of eye movement. Based on these results, it is safe to arrive at a conclusion that the verbs in the main clause might not be able to influence the processing of relative clauses. Yet, they would influence the final result of sight-interpreting because most of the interpreters tend to treat the relative clause as an independent sentence in Chinese.

\section{Conclusion}

Sight-interpreting proves to be typical of conference interpreting in that it requires both the processing of reading and producing almost at the same time. The double endeavor proves to be difficult in interpreting the relative clauses, either SR or OR. With the help of eye movement technology, the results of cognitive efforts of the relative pronoun "who" as the subject and object in the clause are as follows: 1) As far as the processing of antecedents is concerned, interpreters will pay more attention to the antecedent of OR relative clauses. In other words, OR requires more effort as reflected in more regression-in. 2) As far as the processing of relative clauses is concerned, interpreters' cognitive efforts in processing OR relative clauses are greater, which is shown in more regression-out, longer first fixation duration, gaze duration, regression duration, and total reading duration. That is to say, the long distance between the antecedent and its original place in the OR relative clause requires the interpreters to exert more efforts to process the relation. 3) The verbs of the main clause basically have no effect on the measures of sight-interpreting relative clauses of SR and OR structure.

There are, however, two questions that remain unanswered. First, each sentence in the experiment only has an average of 10 words within 4000 most frequent BNC. It is possible that with the increase of the number and less frequency of words, the results of the measures of eye-movement would be different. It is predicted that the longer the sentences are, the closer the time used to process SR and OR relative clauses in sight-interpreting because the length of sentence correlate highly with the interpreter's working memory, which is limited, roughly within 5-9 information blocks [6]. Besides, only two types of relative clauses are explored. Further research can be designed to uncover the cognitive processing of other roles that antecedent plays in its relative clauses such as adverbial, or expand the scope of research to adverbial clauses, appositive clauses and object clauses, so as to provide more empirical research for the interpretation process.

\section{Funding}

This paper was supported by Philosophy and Social Science Research Fund of Hangzhou City (Reference No: M19JC036).

\section{References}

[1] Newmark, Peter. (2001). A Textbook of Translation. Shanghai: Shanghai Foreign Language Education Press.

[2] Yang Chengshu. (2010). On the Process of Information during Interpreting. Tianjin: Nankai University Press.

[3] Zhao Xueqin \& Xu Hanyu. (2018). The Influence of Logical Connectors on the Cognitive Effort during Sight-interpreting from Chinese into English. Foreign Language Research, 5: 7-11.

[4] Blumstein, Sheila E. (2019). Neurolinguistics: A Brief Historical Perspective. In Greig I. De Zubicaray \& Niels O. Schiller (Eds.) The Oxford Handbook of Neurolinguistics (pp. 1-11). New York: OUP.

[5] Wang Jianhua. (2019). Cognition and Interpretation. Beijing: Press of China Renmin University.

[6] Liu Jin. (2017). Research on the Cognitive Processing Mode of Sight-translation. Chinese Interpreters Journal, (2): 25-30.

[7] Xiang Xia \& Zheng Binghan. (2015). Background information and Quality of Interpreting. Foreign Countries and Foreign Language Teaching, 1: 94-102.

[8] Gile, D. (1995). Basic Concepts and Models for Interpretation and Translator Training. Amsterdam: John Benjamins Publishing Company.

[9] Wan Hongyu (2005). Research of Process of Sight-interpreting and its Guideline for Teaching. Unpublish PhD Dissertation, Shanghai Foreign Studies University. 
[10] Deng Wei. (2017). Review and Reflection on the Study of Sight-translation in China over the Past 30 Years. Foreign Language and Literature, 33 (5): 97-102.

[11] Chen Dezhang. (2011). Approach to Translation between English and Chinese. Beijing: Foreign Language Teaching and Research Press.

[12] Wang Jinquan. (2002). A Case Study of English-Chinese Translation of English Attributive Clauses. Foreign Language Teaching and Research, 34 (6): 471-475.

[13] Gibson, E., et al. (2005). Reading Relative Clauses in English. Cognitive Linguistics, 16 (2): 313-353.

[14] Yan Guoli \& Bai Xuejun. (2018) The Base and Application of Eye Movement Analytical Technology. Beijing: Beijing Normal University Press
[15] Fan Lin \& Liu Zhenqian. (2007). Research on Eye Movement of Reading and Understanding Process. Foreign Languages and Their Teaching, 217 (4): 35-40.

[16] Rayner, K. \& Pollatsek, A. (2006). Eye-movement Control in Reading. In M. J. Traxler \& M. A. Gernsbacher (Eds.), Handbook of Psycholinguistics (2nd ed. pp. 613-657). San Diego, CA: Academic Press.

[17] Yan Guoli., et al. (2013). Introduction to the Main Eye Movement Parameters of Research on Reading. Advances in Psychological Science, 21 (4): 589-605.

[18] Zhao Ming \& Liu Tao. (2011). The ERP Research on the Anaphoric Linguistic Processing. Advances in Psychological Science, 19 (3): 355-363.

[19] Yan Guoli \& Bai Xuejun. (2012). General Introduction to the Eye Movement Research. Beijing: Science Press. 